\title{
Metodologías aplicadas en la evaluación de la competencia transversal “Comprensión e Integración" en los estudios universitarios del Grado de Administración y Dirección de Empresas en la Universitat Politècnica de València
}

\author{
Marín-Sánchez, María del Mara; Crespo Abril, Fortunatob; Maroto Álvarez, \\ Concepción $^{\mathrm{b}}$; Palau Ramirez, Felipe ${ }^{\mathrm{c}}$; Palmer Gato, Martad ${ }^{\mathrm{d}}$, Úbeda García, Juan \\ E. ${ }^{\mathbf{e}}$ \\ ${ }^{a}$ CEGEA, Departamento de Economía y Ciencias Sociales, Universitat Politècnica de València, España, \\ mmarins@esp.upv. bepartamento de Estadística e Investigación Operativa, Universitat Politècnica de València, \\ España,. ${ }^{\mathrm{C}}$ Departamento de Economía y Ciencias Sociales, Universitat Politècnica de València, España, ${ }^{\mathrm{d} D e p a r t a m e n t o}$ \\ de Organización de Empresas, Universitat Politècnica de València, España, e'Departamento de Economía y Ciencias \\ Sociales, Universitat Politècnica de València, España
}

\begin{abstract}
In this paper we analyze the implementation of the Transversal Competence "Understanding and integration" through the subjects of Business Administration and Management degree. It is kept in mind that the proficiency level of the competence can not be the same for 1st and 2nd years, which would correspond to level 1, that for 3rd and 4th years, to which they correlate a higher level 2 The broad range of methodologies and techniques used in the assessment of this skill, allows us to infer that the overall evaluation is correct.
\end{abstract}

Keywords: transversal competences, comprehension and integration, competency training, competence assessment.

\section{Resumen}

Las metodologías aplicadas en la implementación de la Competencia Transversal "Comprensión e integración" en los estudios de Administración y Dirección de Empresas, son muy diversas predominando el estudio de casos y la resolución de problemas. En el presente trabajo se analiza cómo se aplican y qué ventajas e inconvenientes se observan. Se concluye que al evaluar al alumno desde distintas perspectivas con diferentes metodologías, el resultado global integra los distintos niveles de dominio de la competencia.

Palabras clave: competencias transversales, comprensión e integración, formación en competencias, evaluación de competencias, administración de empresas. 


\section{Introducción y Objetivos}

La UNESCO, en la Conferencia Mundial sobre Educación Superior de París de 1998, señaló como uno de los ejes prioritarios la formación en competencias (Arroyo-Cañada, 2019), por lo que cada vez son más las Universidades que consideran prioritario la evaluación por competencias. Así, las denominadas Competencias Transversales tienen un indudable valor en el desempeño profesional de los titulados universitarios. En este sentido la Universitat Politècnica de València (UPV) ha puesto en marcha un Proyecto institucional para incluir de forma explícita la exigencia de que nuestros estudiantes se formen en estas competencias y sean evaluados para poder ser acreditado su nivel de dominio.

El objetivo del presente trabajo es comparar las metodologías empleadas en distintas asignaturas que tienen en común la evaluación de la Competencia Transversal "Comprensión e Integración", en la titulación de Grado en Administración y Dirección de Empresas de la UPV y así poder concluir si realmente los alumnos consiguen el nivel de dominio de la competencia.

\section{Contenido del Proyecto Institucional de Competencias Transversales de la Universitat Politècncia de València. La Competencia Transversal "Comprensión e Integración}

El Proyecto Institucional de la UPV tiene como objetivo fundamental (UPV, 2018):

- Establecer la estrategia de evaluación sistemática de las Competencias Transversales, definiendo cómo se adquieren y cómo deben ser evaluadas, y

- Acreditar la adquisición de dichas competencias.

Con este Proyecto institucional se puede afirmar que la UPV podrá:

- Proporcionar a sus egresados un valor añadido que puede diferenciarlos de otros egresados y, en consecuencia, hacer más atractivos los estudios ofertados frente a ofertas similares de otras universidades.

- Poner en valor la capacitación de nuestros egresados de cara a los empleadores.

- Explicitar la adquisición de las competencias de cara a acreditaciones internacionales.

En el ámbito de este Proyectyo, la UPV examinó las Competencias Transversales explicitadas en cada título ofrecido y en base a los mismos se han definido trece Competencias Transversales. La primera de ellas es la que nos ocupa en este trabajo de coordinación: "Comprensión e Integración". Comprender quiere decir "percibir y tener una idea clara de lo que se llama, se hace o sucede, o descubrir el sentido profundo de algo". Para demostrar que algo se ha comprendido, la persona identifica y recupera la información, y lo explica con sus palabras, interpretando e integrando las ideas desde su perspectiva. 
Los tres niveles de dominio que se contemplan son:

Nivel 1: Describir, relacionar e interpretar situaciones y planteamientos sencillos. Se correspondería con asignaturas de $1^{\circ}$ y $2^{\circ}$ de Grado.

Indicadores:

- Identificar correctamente las ideas o conceptos de un texto, problema, ejercicio...

- Explicar correctamente, con sus propias palabras, las ideas planteadas en un texto, problema, ejercicio...

- Interpretar, expresando de manera personal, las ideas o conceptos planteados en un texto, problema, ejercicio...

- Integrar diferentes ideas o conceptos de la asignatura en sus análisis.

Nivel 2: Proceder con lógica y relacionar e integrar conceptos en situaciones complejas. Se correspondería con asignaturas de $3^{\circ}$ y $4^{\circ}$ de Grado

Indicadores:

- Clasificar y establecer relaciones significativas entre diferentes ideas o conceptos.

- Proporcionar sentido a los planteamientos que se le presentan para poder interpretarlos.

- Diferenciar los factores causantes o consecuentes de un hecho.

- Transferir los contenidos a la práctica, integrándolos en un contexto.

- Integrar elementos de diferentes asignaturas o áreas en sus análisis de la realidad.

Nivel 3: Identificar las carencias de información y utilizar el conocimientos con un enfoque globalizador.

Indicadores:

- Identificar lagunas de información o falta de coherencia en las argumentaciones.

- Establecer generalizaciones o relaciones causa-efecto.

- Expresar sus ideas y generar conclusiones, partiendo de diferentes datos y sus relaciones.

- Mostrar una visión sistémica entre varias disciplinas y/o áreas de conocimiento.

Las actividades formativas para el desarrollo de esta competencia implican interpretar información de forma reflexiva y precisa para lo cual se require, entre otras, las siguientes actividades:

- Comparar conceptos, principios y teorías, examinar parecidos y diferencias entre dos o más objetos, ideas, situaciones etc.

- Clasificar: agrupar objetos, sucesos, ideas, etc. según sus características comunes precisando el mejor criterio de agrupación, con el fin de organizar eficazmente el conocimiento y las actividades profesionales y de la vida diaria, etc.

- Analizar y sintetizar. El análisis consiste en aislar los elementos constituyentes del todo, identificándolos, relacionándolos entre sí y con el todo. La síntesis supone 
la integración de todas y algunas partes para producir alguna nueva configuración significativa, alguna conclusión, etc.

- Secuenciar: consiste en ordenar series de elementos que se suceden unos a otros según un criterio que marca la dirección de la progresión.

- Descubrir razones: consiste en indagar las razones ciertas, dudosas e inciertas que sustentan un argumento, una conducta, una información, etc. Evaluando si las mismas apoyan y justifican lo que se desea comunicar y también su solidez, relevancia y suficiencia.

Este tipo de actividades se pueden desarrollar siguiendo metodologías de diferente tipo, como por ejemplo:

- Actividades grupales

- Exposiciones orales

- Foros y debates

- Lecturas

- Prácticas de laboratorio

- Preguntas

- Problemas

- Proyectos

- Redacción de informes

- Seminario

- Entre los posibles procedimientos de evaluación de esta competencia, destacamos:

- One minute paper

- Sondeo

- Pruebas objetivas

- Redacción de informes

- Autoevaluación

- Evaluación entre iguales

- Elaboración de mapas conceptuales

3. Comparativa de las metodologías aplicadas en la consecución de la Competencia Transversal Comprensión e Integración en las asignaturas que son punto de control en el Grado de ADE de la UPV

En el Grado de Administración y Dirección de Empresas son seis las asignaturas en las que se evalúa especialmente la competencia transversal de "Comprensión e Integración", como se muestra en la Tabla 1. 
Tabla 1. Asignaturas en las que se evalúa la CT “Comprensión e Integración”

\begin{tabular}{cc}
\hline Nivel 1 & Nivel 2 \\
\hline Derecho de la Empresa $\left(1^{\circ}\right)$ & Investigación Operativa $\left(3^{\circ}\right)$ \\
Métodos Estadísticos en & Gestiòn Fiscal de la Empresa $\left(3^{\circ}\right)$ \\
Economía $\left(2^{\circ}\right)$ & \\
Métodos Cuantitativos para la \\
Ayuda a la toma de decisiones $\left(3^{\circ}\right)$ \\
Valoración de Empresas $\left(4^{\circ}\right)$
\end{tabular}

\subsection{La asignatura de "Derecho de la Empresa"}

Es una asignatura obligatoria de $1^{\circ}$ curso, por lo que correspondería al Nivel 1 de dominio en la competencia.

El alumno ha de manejar fuentes del derecho y bibliografía y además debe realizar lecturas de noticias relacionadas con los contenidos vistos en cada tema. La nota de exámenes tipo test realizados a lo largo del curso y la nota de los Seminarios es la nota de la Competencia. En resumen:

- Actividad formativa: clases de teoría, lecturas y seminarios.

- Procedimiento de evaluación: pruebas objetivas; redacción de informes a partir de noticias relacionadas con la asignatura.

- Instrumento de evaluación: Se define como criterio, a partir de la nota obtenida en la asignatura. La equivalencia empleada con la nota númerica supone que finalmente la "Excelencia" sería con la letra A, "Adecuado" se define con la letra B, "En Progreso" corresponde a la letra C y "Sin Alcanzar" sería la letra D.:

del siguiente modo:

- $\quad \mathrm{D}:<2,5$ puntos

- C: 2,5-4,9 puntos

- B: $5,00-7,5$

- A: $>7,5$

\subsection{La asignatura "Métodos estadísticos en economía"}

Es una asignatura Obligatoria de $2^{\circ}$, por tanto del nivel 1 de dominio de la competencia.

Durante el desarrollo de las clases magistrales, y en la realización de las siete prácticas de laboratorio, se realizan actividades que exigen la participación del alumno en la resolución de casos prácticos. Las últimas prácticas de laboratorio presentan actividades especialmente diseñadas para fortalecer esta competencia. En resumen:

- Actividad formativa: lección magistral, prácticas de laboratorio, preguntas y problemas 
- Procedimiento de evaluación: Algunas preguntas del examen final se centran en valorar los aspectos relacionados con esta competencia

- Instrumento de evaluación: Nota obtenida en las preguntas del examen que evalúan la competencia. La equivalencia empleada con la nota númerica supone que finalmente la "Excelencia" sería a la letra A, "Adecuado" se define con la letra B, "En Progreso" corresponde a la letra C y "Sin Alcanzar" sería la letra D del siguiente modo:

- $\quad$ D: $<2,5$ puntos

- C: $2,5-4,9$ puntos

- B: $5,00-7,5$

- $\mathrm{A}:>7,5$

\subsection{La asignatura de "Investigación Operativa"}

Es obligatoria de 3er curso, de nivel 2 en lo que se refiere al dominio de la competencia.

Los alumnos realizan 10 casos prácticos en grupos de 2-3 alumnos en el aula informática. Los casos prácticos formulan problemas de toma de decisiones que los alumnos deben abordar mediante el planteamiento y resolución de modelos de optimización/multicriterio, cuyos resultados se interpretan para tomar decisiones concretas. Este trabajo se presenta en informes escritos y/o juegos (Kahoot).

En resumen

- Actividad formativa: actividades grupales; prácticas informáticas, casos

- Procedimiento de evaluación: redacción de informe escrito y juegos (Kahoot).

- Instrumento de evaluación: Nota obtenida en las prácticas y en la asignatura al estar ambas relacionada. La equivalencia empleada con la nota númerica supone que finalmente la "Excelencia" sería a la letra A, "Adecuado" se define con la letra B, "En Progreso" corresponde a la letra C y "Sin Alcanzar" sería la letra D:

- D: $<2,5$ puntos

- C: 2,5-4,9 puntos

- B: $5,00-7,5$

- $\mathrm{A}:>7,5$

\subsection{La asignatura de "Gestión Fiscal de la Empresa"}

Es obligatoria de 3 er curso, por tanto de nivel 2 en el dominio de la competencia.

Se realizan seis prácticas de laboratorio en las que se resuelve un caso con ayuda del Programa Informático de ayuda de la Agencia Tributaria española en el que los alumnos pueden revisar el modelo oficial de presentación del Impuesto y el cual deben completar con la solución del caso. De ese modo integran los conocimientos adquiridos al tener que decidir cuál es la mejor manera de calcular la liquidación del impuesto. La evaluación del correcto desempeño se realiza a través de un examen tipo test on line empleando la 
herramienta de la intranet, Poliformat. La nota media en dichas prácticas sirve para la calificación de la competencia transversal.

- Actividad formativa: Prácticas de laboratorio

- Procedimiento de evaluación: Pruebas de respuesta corta y Pruebas de ejecución.

- Instrumento de evaluación: Nota obtenida en las prácticas de laboratorio. La equivalencia empleada con la nota númerica supone que la "Excelencia" sería la letra A, "Adecuado" se define con la letra B, "En Progreso" corresponde a la letra C y "Sin Alcanzar" sería la letra D.

- D: $<2,5$ puntos

- C: $2,5-4,9$ puntos

- B: $5,00-7,5$

- A: $>7,5$

\subsection{La asignatura de "Métodos cuantitativos para la Ayuda a la Toma de Decisiones"}

Es obligatoria de 3er curso, por tanto de nivel 2 en el dominio de la competencia.

Los alumnos resuelven 1 caso práctico, en grupos de como máximo 4 participantes, en el que deben analizar una situación utilizando todos los conocimientos de los que dispongan y resolver argumentando sus decisiones

- Actividad formativa: actividades grupales; casos.

- Procedimiento de evaluación: redacción de informe escrito.

- Instrumento de evaluación: a través de la observación y correlacionándolo con la nota global de la asignatura

\subsection{La asignatura de "Valoración de Empresas"}

Es optativa de $4^{\circ}$ curso, por tanto de nivel 2 en el dominio de la competencia.

Se elabora un trabajo final en el que se analizan las metodologías explicadas en clase integrándolas en un trabajo final.

- Actividad formativa: casos

- Procedimiento de evaluación: Redacción de informe.

- Instrumento de evaluación: nota obtenida en las prácticas de laboratorio. La equivalencia empleada con la nota númerica supone que finalmente la "Excelencia" sería a la letra A, "Adecuado" se define con la letra B, "En Progreso" corresponde a la letra C y "Sin Alcanzar" sería la letra D.

- D: $<2,5$ puntos

- C: 2,5-4,9 puntos

- B: $5,00-7,5$

- $\mathrm{A}:>7,5$ 


\section{Ventajas e Inconvenientes observados en la comparativa de las distintas metodologías}

La principal ventaja que se observa es la diversidad y variedad en las actividades formativas empleadas en cada asignatura para evaluar la competencia transversal, si bien es cierto que predomina la utilización del método del caso. Esto es muy positivo porque implica que la evaluación de la Competencia es efectiva y así se asegura su consecución por parte del estudiante.

Asimismo los procedimientos de evaluación son también distintos, adecuándose a las actividades formativas realizadas en cada asignatura, lo cual entendemos redunda en la calidad docente finalmente alcanzada.

En lo que se refiere a los inconvenientes podemos destacar que en la evaluación de actos grupales es necesario completarlo con información individualizada ya sea mediante la observación o con preguntas dirigidas en el examen.

También cabe señalar que la gran cantidad de profesorado asociado (un 46\% de los profesores que imparten su docencia en los estudios del Grado de ADE son Asociados a tiempo parcial), dificulta la correcta implementación de las distintas actividades programadas para asegurar que su ejecución es igual en todos los grupos, generándose a veces diferencias difíciles de superar.

\section{Conclusiones}

La Competencia Transversal "Comprensión e Integración" es relativamente sencilla de implementar y evaluar dado que está en el corazón de la docencia al formar parte de los objetivos que se persiguen. No obstante es necesaria la coordinación entre los profesores que están evaluando la misma Competencia para poder verificar si de forma continuada los procedimientos de evaluación están detectando la adquisición de la competencia en los dos niveles de dominio que manejamos en el Grado de Administración y Dirección de Empresas.

La variedad y complementariedad de actividades formativas y de procedimientos de evaluación, confirman que la Competencia se adquiere y que se puede determinar su adquisición por el estudiante de forma satisfactoria.

Finalmente, se considera que disponer de un criterio homogéneo de evaluación permite aclarar a los alumnos los resultados obtenidos dotándole de un sistema transparente de evaluación, y no generar dudas y posibles reclamaciones.

De este modo el valor añadido para este Grado se incrementa de forma sustancial al poder ser contrastable el nivel de dominio de las Competencias Transversales al realizarse su evaluación de forma sistemática 


\section{Referencias}

ANECA (2019). Guía de apoyo para la redacción, puesta en práctica y evaluación de los resultados del aprendizaje. Versión 1.0. ANECA. $<$ http://www.aneca.es/Documentosy-publicaciones $>$ [Consulta:14 mayo 2019].

ARROYO-CAÑADA, F-J. (2019). "La evaluación por competencias en estudios empresariales" en Journal of Management and Business Education, vol 2(1), p. 1-7

DELORS, J. (1996). La educación encierra un tesoro. Informe a la Unesco de la Comisión Internacional sobre la Educación para el siglo XXI. $<$ http://unesdoc.unesco.org/images/0010/001095/109590so.pdf $>$ [Consulta 14 mayo 2019].

UPV, (2019). Proyecto institucional de las competencias transversales UPV. $<$ http://www.upv.es/competenciastransversales> [Consulta 1 junio 2019] 